\title{
Visible and near-infrared bulk optical properties of raw milk
}

\author{
B. Aernouts, ${ }^{1}$ R. Van Beers, R. Watté, T. Huybrechts, J. Lammertyn, and W. Saeys \\ KU Leuven, Department of Biosystems, MeBioS, Kasteelpark Arenberg 30, 3001 Leuven, Belgium
}

\begin{abstract}
The implementation of optical sensor technology to monitor the milk quality on dairy farms and milk processing plants would support the early detection of altering production processes. Basic visible and nearinfrared spectroscopy is already widely used to measure the composition of agricultural and food products. However, to obtain maximal performance, the design of such optical sensors should be optimized with regard to the optical properties of the samples to be measured. Therefore, the aim of this study was to determine the visible and near-infrared bulk absorption coefficient, bulk scattering coefficient, and scattering anisotropy spectra for a diverse set of raw milk samples originating from individual cow milkings, representing the milk variability present on dairy farms. Accordingly, this database of bulk optical properties can be used in future simulation studies to efficiently optimize and validate the design of an optical milk quality sensor. In a next step of the current study, the relation between the obtained bulk optical properties and milk quality properties was analyzed in detail. The bulk absorption coefficient spectra were found to mainly contain information on the water, fat, and casein content, whereas the bulk scattering coefficient spectra were found to be primarily influenced by the quantity and the size of the fat globules. Moreover, a strong positive correlation $(\mathrm{r} \geq 0.975)$ was found between the fat content in raw milk and the measured bulk scattering coefficients in the 1,300 to $1,400 \mathrm{~nm}$ wavelength range. Relative to the bulk scattering coefficient, the variability on the scattering anisotropy factor was found to be limited. This is because the milk scattering anisotropy is nearly independent of the fat globule and casein micelle quantity, while it is mainly determined by the size of the fat globules. As this study shows high correlations between the sample's bulk optical properties and the milk composition and fat globule size, a sensor that allows for robust separation between the absorption and scatter-
\end{abstract}

Received March 26, 2015.

Accepted June 4, 2015.

${ }^{1}$ Corresponding author: Ben.Aernouts@kuleuven.be ing properties would enable accurate prediction of the raw milk quality parameters.

Key words: milk fat globule, casein micelle, visible and near-infrared spectroscopy, absorption, optical sensor design

\section{INTRODUCTION}

A precondition for increased profitability in dairy farming is an increase in both the lactation and lifetime production per cow. Therefore, more effective prevention and early treatment of all diseases, especially the so-called production diseases, are needed (Hamann and Krömker, 1997). To meet these demands, individual cow and udder health should be carefully monitored. Because milk production is a dominant factor in the metabolism of dairy cows, involving a very intensive interaction with the blood circulation, the extracted milk contains valuable information on the nutritional, metabolic, and infectious status of the cow (Hamann and Krömker, 1997; Mulligan et al., 2006; Friggens et al., 2007; Forsbäck et al., 2009; Løvendahl et al., 2010; Aernouts et al., 2011). Therefore, regular analysis of the produced milk is considered to be the most efficient way to monitor cow and udder health. Online measurement of the milk components (fat, protein, lactose, and so on) during milking twice a day would offer the potential for early detection of systemic and local alteration, thus providing a valuable input for strategic and operational management decisions (Friggens et al., 2007).

Visible (Vis) and near-infrared (NIR) spectroscopic analysis of raw milk allows for a reliable detection of the fat, protein, and lactose concentration in the laboratory (Aernouts et al., 2011). The prediction of this milk composition is mainly based on the wavelengthdependent absorption of Vis/NIR radiation by the milk constituents. The industry has recently adopted this technology and implemented it into milking systems to measure the major milk components online (Katz et al., 2011, 2003; Pinsky et al., 2013). However, despite the continuous recalibration, their accuracy and robustness is still not sufficient to support cow health management (Kaniyamattam and De Vries, 2014). This is mainly because the measured spectral signals are, next to absorp- 
tion, considerably influenced by the physical properties of the milk in terms of the quantity and size of the fat globules and $\mathrm{CN}$ micelles. Because the refractive indices of milk fat and $\mathrm{CN}$ differ from that of the milk serum, the Vis/NIR radiation is forced to deviate from its straight trajectory (Tuchin, 2007). Because of these scattering processes, the traveling path of the radiation increases to an unknown extent. This complicates the prediction of the composition from measured spectra. Homogenization of the milk fat globules could reduce and standardize the Vis/NIR scattering to improve the prediction results. For example, in the early days of milk analysis, the Milko-tester (Foss Electric, Hillerød, Denmark) measured the Vis scattering after dispersion of the $\mathrm{CN}$ micelles and homogenization to produce a more uniform fat globule size distribution. Accordingly, the light attenuation depends mainly on the amount of fat globules and can, therefore, be used to obtain a rough estimate for the fat content (McDowell, 1968). However, because of its destructive character, high energy consumption, and significant wear and tear, the proceeding homogenization step is not desired in online analyses on farm. Alternatively, the nonlinear interference due to light scattering can be reduced with empirical methods (e.g., baseline correction, derivatives, and so on) or oversimplistic scattering models (e.g., normal, piecewise, and extended multiplicative scatter correction, path length correction method, and so on), and can be partially accounted for by the prediction models (e.g., partial least squares, support vector machines, and so on; Aernouts et al., 2011). However, these techniques only provide acceptable results for samples with absorption and scattering properties similar to those consulted in the calibration procedure (Melfsen et al., 2013). As the quantity and size of the fat globules and $\mathrm{CN}$ micelles for different fresh raw milk samples experience large variations, the scattering properties also vary a lot (Vangroenweghe et al., 2002; Nielsen et al., 2005; Cabassi et al., 2013; Logan et al., 2014). Consequently, it is very challenging to fully compensate for all this scattering variability with a single empirical calibration model. Therefore, more powerful and advanced techniques are needed to remove the scattering interference from the measured Vis/NIR spectra (Melfsen et al., 2012).

In Vis/NIR spectroscopy, accurate separation of the absorption and scattering properties would reduce the need for empirical scatter corrections and promote robust prediction of the sample composition (Steponavičius and Thennadil, 2009, 2011, 2013). Moreover, the pure absorption, defined as the bulk absorption coefficient $\boldsymbol{\mu}_{\boldsymbol{a}}\left(\mathrm{cm}^{-1}\right)$, is the probability of absorption per unit infinitesimal path length at a specific radiation wavelength and relates directly to the sample composition according to the Beer-Lambert law. The scattering, on the other hand, can be described with the bulk scattering coefficient $\boldsymbol{\mu}_{s}\left(\mathrm{~cm}^{-1}\right)$ and the angular scattering pattern or scattering phase function. The bulk scattering coefficient defines the probability of scattering per unit infinitesimal path length in a similar way as $\mu_{a}$ represents the absorption. The scattering phase function is generally too complex to reproduce and interpret and is, therefore, often represented by its mean cosine: the scattering anisotropy factor $\boldsymbol{g}$. The scattering anisotropy for biological tissues and fluids in the Vis/NIR range varies between 0 (isotropic scattering) and 1 (complete forward scattering; Tuchin, 2007). These scattering properties are determined by the physical microstructure properties of the sample (e.g., particle size distribution, particle volume concentration, material properties, and so on). For milk, this primarily relates to the quantity and size of the suspended fat globules and, to a smaller extent, the CN micelles (Bogomolov et al., 2012, 2013; Bogomolov and Melenteva, 2013; Dahm, 2013; Kucheryavskiy et al., 2014; Aernouts et al., 2015). As these properties affect the physicochemical, functional, and sensory characteristics of the raw milk and derived dairy products, they are important quality parameters (Michalski et al., 2003, 2004; Walstra et al., 2006; Cabassi et al., 2013; Schenkel et al., 2013). Moreover, the size of fat globules in milk from infected udder quarters (mastitis) is increased significantly and could, therefore, give insight into the udder health status of each individual cow and udder quarter (Erwin and Randolph, 1975; Mizuno et al., 2012). Accordingly, extraction of physical microstructure information, such as the fat globule size distribution, from isolated scattering properties would create an added value for Vis/NIR spectroscopy on raw milk (Cabassi et al., 2013; Aernouts et al., 2015).

In a single Vis/NIR spectroscopic measurement, usually reflectance or transmittance, both the effect of absorption by the chemical molecules and scattering by the physical particles are interconnected and cannot be accurately separated. Consequently, a change in the scattering properties of a measured milk sample might be misinterpreted as a change in the milk composition (Melfsen et al., 2012). On the other hand, multiple spectroscopic measurements in a slightly different configuration are not perfectly correlated and will, therefore, be influenced by absorption and scattering in a different way. The combination of such multiple measurement series with an accurate model, which mathematically describes light propagation as a function of the sample's bulk optical properties $\left(\mu_{a}, \mu_{s}\right.$, and $g$ ), could provide a successful separation of the sample's absorption and scattering properties (Steponavičius and Thennadil, 2013). However, superior separation between these ab- 
sorption and scattering properties is only feasible if the optical sensor is designed to obtain a series of multiple measurements with least intercorrelation and maximum signal-to-noise levels. As the measured signals are, next to the sensor architecture, determined by the sample's bulk optical properties (BOP), the optimal design of such a practical sensor configuration depends on the absorption and scattering properties of the samples to be measured. The effect of the BOP on the light propagation, and consequently the collected spectral signals, is very complex. Therefore, the optimal design cannot be calculated directly from a supplied range of $\mathrm{BOP}$, though it can be determined through an iterative optimization procedure. In practice, a wide range of sensor configurations is physically possible. So, it is preferred to test the potential of each sensor configuration through these simulations, rather than building each of them and evaluating their performance from measurements on an extensive set of raw milk samples (Luo et al., 2005; Liu and Ramanujam, 2006; Sharma et al., 2006; Palmer and Ramanujam, 2007; Cen et al., 2010; Gamm et al., 2011; Khankin et al., 2012; Zamora-Rojas et al., 2014). The sensor configuration, which allows for the most robust separation between the absorption and scattering properties, would obviously have the highest potential to retrieve accurate predictions for the milk composition (fat, protein, lactose, urea, and so on) and physical properties (fat globule and $\mathrm{CN}$ micelle size distribution) from, respectively, the obtained absorption and scattering properties. As these milk quality properties are highly correlated with cow health, such sensor would support on-farm dairy management.

The Monte Carlo method for simulation of light propagation is very accurate, flexible, and widely used in tissue optics (Tuchin, 2007). Therefore, it is particularly suitable to simulate the collected spectra series for each sensor configuration and for the range of BOP found in raw milk samples. Accordingly, the potential of each sensor configuration can be defined as the ability to extract the BOP from the collected signals, after adding noise typical for a Vis/NIR spectrometer. Such procedures of sensor design optimization have been widely studied and improved in the last decade and are still an important topic of research (Luo et al., 2005; Liu and Ramanujam, 2006; Sharma et al., 2006; Palmer and Ramanujam, 2007; Cen et al., 2010; Gamm et al., 2011; Khankin et al., 2012; Zamora-Rojas et al., 2014). However, to consult these algorithms to obtain an optimal sensor design for quality control of raw milk, knowledge on the Vis/NIR BOP of raw milk is crucial.

Recently, the influence of a varying fat globules size on the Vis/NIR scattering properties of milk was studied in detail (Aernouts et al., 2015). Moreover, reduction of the fat globule size resulted in a higher wavelength- dependency of both the bulk scattering coefficient and the scattering anisotropy factor, reducing their values for wavelengths above $600 \mathrm{~nm}$ and approaching the Rayleigh scattering phenomenon. Nevertheless, to our knowledge, no accurate information is available in literature on the mean, variability, and range of the Vis/ NIR BOP of raw milk from dairy cows, which is therefore defined as the main objective of this study. The measurements focus on the 550 to 1,900 nm wavelength range as, below $550 \mathrm{~nm}$, the main milk components have no relevant absorption peaks, whereas above 1,900 $\mathrm{nm}$, water is a very strong absorber resulting in very low signal-to-noise levels for any type of optical measurement. Next, the obtained data were used to closely study the relation between the Vis/NIR BOP and the milk's chemical and physical quality properties.

\section{MATERIALS AND METHODS}

\section{Milk Samples}

The milk samples considered in this research were collected in the context of the milk production registration system within Flanders (Belgium). Dairy farmers from all over Flanders can participate in this system to monitor the milk composition and production of their individual cows every 4 to 6 wk. These data are used to improve breeding and genetic selection and to some extent for the evaluation and basic adjustment of feeding. For each cow, a representative milk sample $(27 \mathrm{~mL})$ is collected, preserved $\left(4^{\circ} \mathrm{C}\right.$ and $\pm 0.11 \% \mathrm{vol} /$ vol preservative; Qlip N.V., Leusden, the Netherlands), and analyzed with the Milkoscan FT+ (Foss A/S, Hillerød, Denmark) to determine the milk fat and CP content (ISO 9622; ISO, 2000). According to the fabricant, the preservative contained Patent Blue $\mathrm{V}$ calcium salt (CAS: 3536-49-0, Sigma-Aldrich, St. Louis, MO) as a visible colorant marker. For this study, 60 raw milk samples, originating from 60 different cows and 17 different dairy farms, were selected from a large collection of 1,200 samples (20 dairy farms) to cover the maximum range of the compositional variance. Moreover, the 1,200 samples were ordered on fat content, and the first and every 40th sample was selected (total 31 samples). The same procedure was repeated for the protein content on the remaining samples.

Table 1 gives an overview of the most important statistical parameters describing the fat and $\mathrm{CP}$ content of the selected sample set. The $\mathrm{CN}$ content was calculated as $75.5 \%$ of the CP content (Walstra et al., 2006; Aernouts et al., 2015). Comparison of the mean, standard deviation, and range (maximum-minimum) of this sample set (Table 1) with the same statistical parameters of a much larger data set (Milk Control 
Table 1. Basic statistics (all \% vol/vol) on the main components in the 60 consulted raw milk samples

\begin{tabular}{lcccc}
\hline Component & Mean & SD & Minimum & Maximum \\
\hline Fat & 5.24 & 2.01 & 1.52 & 12.0 \\
CP & 2.72 & 0.424 & 2.09 & 3.73 \\
CN & 2.05 & 0.424 & 1.58 & 2.82 \\
\hline
\end{tabular}

Center-Flanders) indicates that the 60 samples are representative for the large population of milk produced by individual cows in Flanders (Aernouts et al., 2011). The correlation between both components was found to be in the normal range $(\mathrm{r}=0.34$; Aernouts et al., 2011).

\section{Measurement of Bulk Optical Properties for Raw Milk Samples}

Double integrating sphere and unscattered transmittance measurements were used to determine the BOP of the milk samples, as this is considered to be the gold standard method for BOP measurement of thin samples of turbid media. The sample illumination in this setup was especially designed to obtain high signal-to-noise spectra in the 500 to $2,250 \mathrm{~nm}$ wavelength range for very turbid media such as raw milk. It consists of a supercontinuum laser light source $(500-2,250 \mathrm{~nm}, 4 \mathrm{~W}$ optical power) in combination with a high-precision monochromator. The total reflectance $\left(\boldsymbol{M}_{\boldsymbol{R}}\right)$ and total transmittance $\left(\boldsymbol{M}_{T}\right)$ were measured simultaneously on each milk sample loaded in a cuvette (Schott, Germany) with a path length of $600 \mu \mathrm{m}$ and positioned between the 2 integrating spheres. Both spheres were equipped with a Vis (400-1100 nm) and NIR (1100-2400 nm) detector. Unscattered transmittance $\left(\boldsymbol{M}_{\boldsymbol{U}}\right)$ was measured in a separate path with the Vis and NIR detectors positioned $1.5 \mathrm{~m}$ behind the sample to limit the fraction of scattered photons collected by the detectors (Aernouts et al., 2013, 2014). To obtain sufficient unscattered transmittance signal, the sample was loaded in a thinner cuvette with a path length of $170 \mu \mathrm{m}$ (Schott, Germany). A series of slits between sample and detector further reduced the number of scattered photons captured in the unscattered transmittance signal. For a more extensive description of the measurement setup, the calibration and measurement procedure and a thorough validation, the reader is referred to Aernouts et al. (2013). Moreover, this validation study showed the high repeatability and signal-noise ratio of the system to obtain the BOP of very turbid samples in the Vis/NIR (Aernouts et al., 2013). The samples were thoroughly stirred before they were measured at $22 \pm 1^{\circ} \mathrm{C}$ (room temperature) to ensure the homogeneity and temperature stability of the sample during the measurement.
All sample spectra were measured from 550 until 1,900 $\mathrm{nm}$ in steps of $10 \mathrm{~nm}$ by automated scanning of the predispersive monochromator. The measurement takes $110 \mathrm{~s}$, which was well below the time span (10 min) after which creaming was starting to have a measurable effect on the collected signals.

The diffuse reflectance $\left(M_{R}\right)$ of the samples was derived from the total reflectance after subtraction of the specular reflectance. The latter was calculated at the air-cuvette and cuvette-sample interfaces through the Fresnel equations, which use the real refractive indices of air (1), the cuvette windows (provided by the manufacturer, Schott, Mainz, Germany), and the milk sample. The refractive index was calculated for each sample individually from the available milk composition data, with the equation proposed by Walstra and Jenness (1984), taking into account the sample temperature $\left(22^{\circ} \mathrm{C}\right)$.

The inverse adding doubling (IAD) routine developed and optimized by Prahl (2010) was consulted to obtain the Vis/NIR BOP spectra from the obtained diffuse reflectance and total and unscattered transmittance spectra. Because of significant contribution of scattered photons, no BOP estimation could be established if the unscattered transmittance was below $0.01 \%$. This was the case for approximately one-third of the samples, mainly for radiation wavelengths shorter than 1,200 $\mathrm{nm}$. If $M_{U}$ was below $0.01 \%$, this measurement was neglected and an estimate for the anisotropy factor $g$ was provided to the IAD algorithm to allow for the separation of $\mu_{a}$ and $\mu_{s}$ (Prahl, 2010). For these samples, the average $g$ spectrum was used as an estimate. Moreover, as the variability between the obtained $g$ spectra was very small [Figure $1(\mathrm{c})$ ], the average $g$ spectrum is expected to be close to the actual $g$ spectrum and the separation between scattering and absorption should be sufficiently accurate (Prahl, 2010). Additionally, also the reduced scattering coefficient $\mu_{s}^{\prime}$ is reported. The $\mu_{s}^{\prime}$ combines $\mu_{s}$ and $g$ according to the similarity relation $\mu_{s}^{\prime}=\mu_{s}(1-g)$ and can be used to accurately describe scattering after sufficient scattering events. In other words, after diffusion of the light, scattering can be accurately described with $\mu_{s}^{\prime}$ alone, without the need for separation between $\mu_{s}$ and $g$ (Tuchin, 2007).

\section{RESULTS AND DISCUSSION}

\section{Variability in the Bulk Optical Properties of Raw Milk}

The BOP spectra for all 60 raw milk samples were extracted from the measured $M_{R}, M_{T}$, and $M_{U}$ spectra with the IAD algorithm. In Figure 1, the mean, mean \pm standard deviation, minimum, and maximum values are shown for the derived BOP. The $\mu_{a}$ spectra [Figure 
(a)

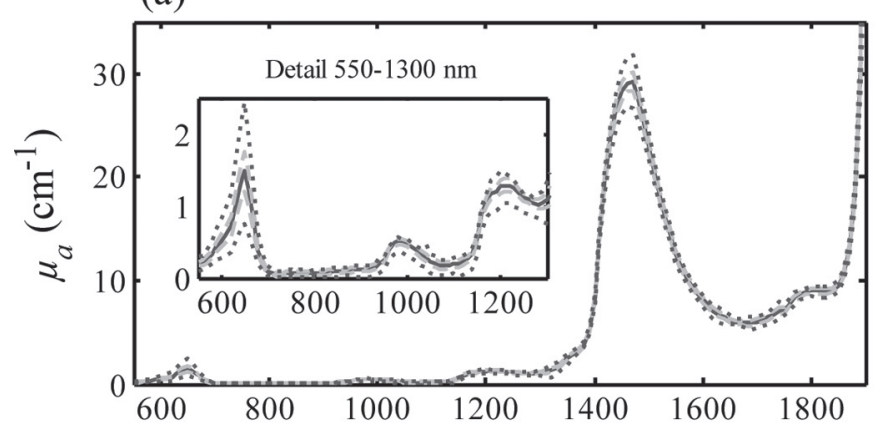

(c)

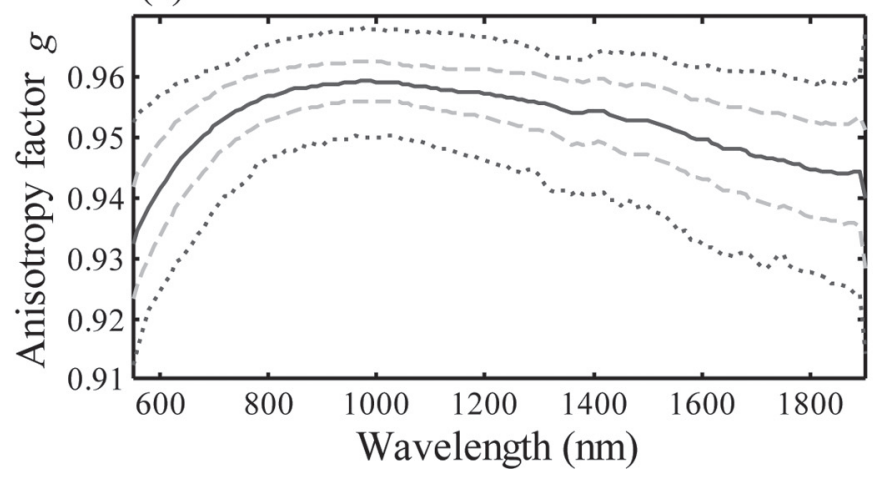

(b)

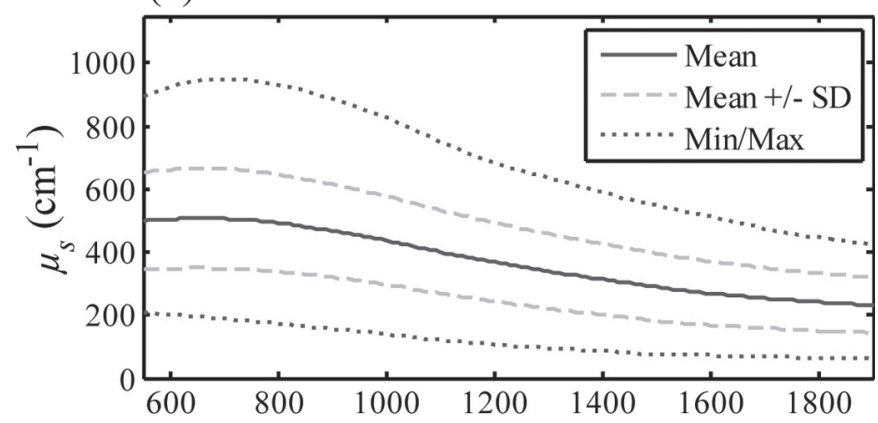

(d)

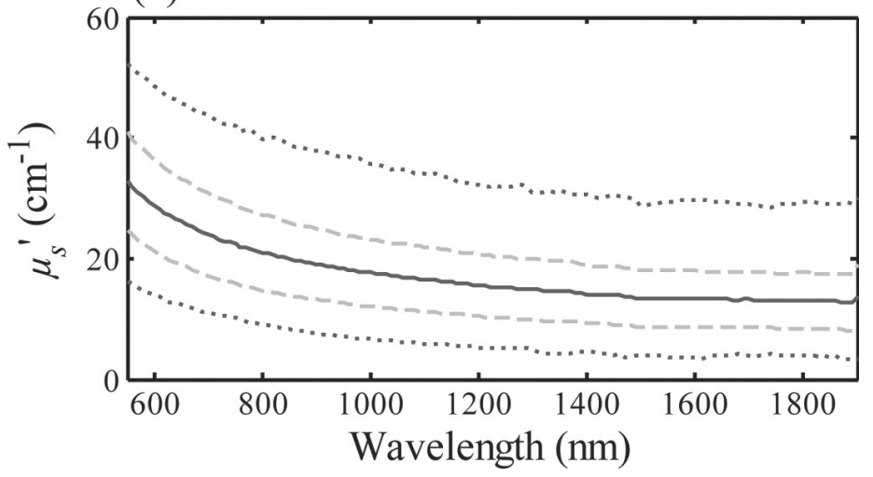

Figure 1. The mean, mean \pm standard deviation, minimum (Min), and maximum (Max) values for the bulk optical properties for 60 raw milk samples in the 550 to $1,900 \mathrm{~nm}$ wavelength range: (a) bulk absorption coefficient $\mu_{a}$, (b) bulk scattering coefficient $\mu_{s}$, (c) anisotropy factor $g$, and (d) reduced scattering coefficient $\mu_{s}^{\prime}$.

$1(\mathrm{a})]$ indicate a very clear signature of water, with absorption peaks around 970, 1,200, 1,450, and 1,940 nm. It should be noted that the peak in $\mu_{a}$ around $650 \mathrm{~nm}$ is caused by the colorant (Patent Blue V), present in the added preservative. Most of the variation in the $\mu_{a}$ spectra can be noticed at the absorption peaks of the colorant, water, and around 1,220 and 1,740 to $1,770 \mathrm{~nm}$. The latter wavelengths are typical absorption peaks for milk fat as they correspond to, respectively, the second and first overtone stretch-vibrations of the $\mathrm{CH}_{2}$ bonds (Šašić and Ozaki, 2000). As the milk fat content varies between 1.52 and $12.0 \%$ (vol/vol; Table 1), noticeable variation can be expected at those absorption bands. Moreover, because of the water displacement effect, a higher DM content, related to a higher fat or CP content, or both, would result in a lower absorption at the water peaks, explaining the considerable variation at the water absorption peaks. Additionally, the high variability around $650 \mathrm{~nm}$ indicates that the preservative concentration clearly varies between samples. At wavelengths where almost no absorption is expected (720-820 nm), still a small baseline of maximum 0.116 $\mathrm{cm}^{-1}$ can be noticed. This is probably the result of very little cross talk between $\mu_{a}$ and $\mu_{s}$ in the BOP estimation procedure. As $\mu_{s}$ is relatively high (100-1,000 $\left.\mathrm{cm}^{-1}\right)$ compared with $\mu_{a}\left(0-35 \mathrm{~cm}^{-1}\right)$, little cross talk of $\mu_{s}$ to $\mu_{a}$ is already noticeable as a small baseline, especially at wavelengths where $\mu_{a}$ is close to zero.

The variation in the scattering coefficient spectra of raw milk in the 550 to $1,900 \mathrm{~nm}$ wavelength range is large, ranging from 100 until almost $1,000 \mathrm{~cm}^{-1}$ [Figure 1(b)]. Fat globules, and to a smaller extent also $\mathrm{CN}$ micelles, are the main cause of scattering in milk (Aernouts et al., 2015). As a result, a higher fat and CN ( p protein) content, which is associated with a higher quantity of respectively fat globules and $\mathrm{CN}$ micelles, results in an increase of the bulk scattering coefficient spectra (Aernouts et al., 2014). Moreover, if scattering events are uncorrelated and the size of fat globules and $\mathrm{CN}$ micelles is stable, a linear positive (independent scattering) relation is present between $\mu_{s}$ and particle quantity (Alexander et al., 2002; Gaygadzhiev et al., 2008; Aernouts et al., 2014). As the variation in fat and protein content in the set of 60 samples is large, it is expected to be the main source of variation in the $\mu_{s}$ spectra. Additionally, as found in an earlier study, the variability in the fat globule size distribution between milk samples will also have an important share in the $\mu_{s}$ variability, especially for wavelengths from 550 until 1,100 nm (Aernouts et al., 2015). Moreover, smaller fat 
globules were found to result in a more steep Vis/NIR $\mu_{s}$ spectrum, with the maximum shifted toward smaller radiation wavelengths and vice versa (Aernouts et al., 2015). The $\mu_{s}$ variation in the set of 60 samples was found to be maximal in the 550 to $1,100 \mathrm{~nm}$ wavelength range, with values ranging from 120 until $950 \mathrm{~cm}^{-1}$. This is probably because the effect of the fat globule size on $\mu_{s}$ is maximal in this wavelength range, additional to the effect of the fat and protein content.

If the independent scattering condition is valid, the anisotropy spectrum should be mainly influenced by the size of the fat globules, while being independent of the fat content itself (Aernouts et al., 2014, 2015). As a result, the variability in the $g$ spectra is relatively small [Figure 1(c)]. In the 550 to $1,900 \mathrm{~nm}$ wavelength range, the anisotropy factor for raw milk increases steadily with increasing radiation wavelength until it reaches a maximum around 1,000 nm (Aernouts et al., 2015). Around these wavelengths, the scattering anisotropy is maximal and the fat globules in raw milk scatter most of the light in the forward direction. For longer radiation wavelengths, the anisotropy factor decreases with increasing wavelength, indicating more isotropic scattering. In a previous study, it was found that a higher $g$ spectrum, mainly for radiation wavelengths above 1,100 $\mathrm{nm}$, indicates larger milk fat globules (Aernouts et al., 2015).

As the reduced scattering coefficient spectrum is the result of both the $\mu_{s}$ and $g$ spectrum, it contains information from both the milk fat globules size and quantity. However, as all the $\mu_{s}^{\prime}$ spectra are almost parallel [Figure 1(d)], it seems that the effects of the fat globule size on $\mu_{s}$ and $g$ neutralize each other if they are combined. In the Vis/NIR, $\mu_{s}^{\prime}$ follows a steady decrease with increasing radiation wavelength until it reaches a nearly stable level for wavelengths above 1,500 $\mathrm{nm}$.

\section{Effect of Fat Globules on Bulk Scattering Properties of Raw Milk}

In earlier studies (Frisvad et al., 2007; Aernouts et al., 2015), it was shown that the fat globules are, next to the CN micelles, the main source of Vis/NIR scattering in unskim milk. This is because the volume fraction of the fat globules is usually larger, and the Vis/NIR scattering intensities for a normalized volume fraction are also higher (Aernouts et al., 2015). Additionally, relative to the fat content, the $\mathrm{CP}$, which consists of $\pm 75.5 \%$ wt/wt CN, experiences only small variations in individual raw milk samples (Table 1; Walstra et al., 2006; Aernouts et al., 2015). As a result, the variability in the bulk scattering properties of raw unskim milk is mainly determined by the variability in the size and quantity of the fat globules. Because of this, the relation between the fat globule size and quantity, and the bulk scattering properties of raw milk, is discussed more in detail. In Figure 2 the bulk scattering properties are shown for 6 raw milk samples with a varying fat content and a practically constant CP content (2.36-2.49\% vol/vol). The CP content was kept constant to further reduce the effect of $\mathrm{CN}$ micelles on the interpreted results. In this plot, 3 groups of each 2 samples can be distinguished based on the fat content, with a large variability between groups and practically no variability within a group. This allows us to study the effect of the fat content and fat globule size separately. The $\mu_{s}$ spectra [Figure 2(a)] indicate that a higher fat content generally results in higher Vis/NIR bulk scattering coefficients. However, within a group of similar fat content, large variability can still be noticed in the $\mu_{s}$ spectra, especially for wavelengths below $1,100 \mathrm{~nm}$. This is probably caused by a difference in size of the fat globules between the 2 samples in the same group. Within the low-fat group (3.45 $\pm 0.065 \%$ $\mathrm{vol} / \mathrm{vol}$ ), small differences between the $\mu_{s}$ spectra can only be noticed for the wavelengths below 1,000 nm. The sample with $3.51 \%$ (vol/vol) fat probably contains slightly smaller fat globules, as an earlier study showed that smaller milk fat globules are related to a steeper $\mu_{s}$ spectrum in the 550 to $1,900 \mathrm{~nm}$ range (Aernouts et al., 2015). The same phenomenon is even more clear for the other 2 groups. Additionally, in these groups, a steeper $\mu_{s}$ spectrum in the Vis/NIR range is also related to a maximum $\mu_{s}$ at smaller wavelengths, typical for smaller scattering particles (Cattaneo et al., 2009; Cabassi et al., 2013; Aernouts et al., 2015). Within each group of raw milk samples with a similar fat content, the $\mu_{s}$ spectra seem to cross each other in the 1,200 to $1,400 \mathrm{~nm}$ wavelength range. Accordingly, the $\mu_{s}$ in this wavelength region might be less dependent on the fat globule size and have a higher correlation with the fat content itself.

As the anisotropy factor should be independent of the fat content if scattering processes are independent, $g$ mainly contains information on the size of the fat globules (Aernouts et al., 2014, 2015). Moreover, an earlier study indicated that larger milk fat globules resulted in a higher anisotropy factor in the 1,100 to $1,900 \mathrm{~nm}$ wavelength range (Aernouts et al., 2015). Within each group, the sample with the smallest fat globules, according to the $\mu_{s}$ spectra (steeper and maximum shifted toward smaller wavelengths), was also characterized by a lower $g$ spectrum for wavelengths above $1100 \mathrm{~nm}$. Moreover, even between groups, a lower $g$ spectrum in the 1,100 to $1,900 \mathrm{~nm}$ wavelength range strongly correlates with a steeper $\mu_{s}$ spectrum and a maximum $\mu_{s}$ at shorter wavelengths. This strengthens the hypotheses that were generated in the previous paragraph. 
(a)

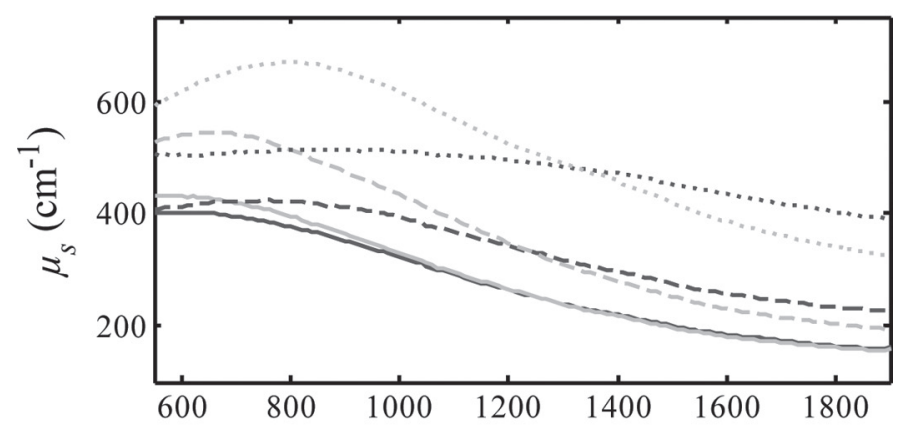

(b)

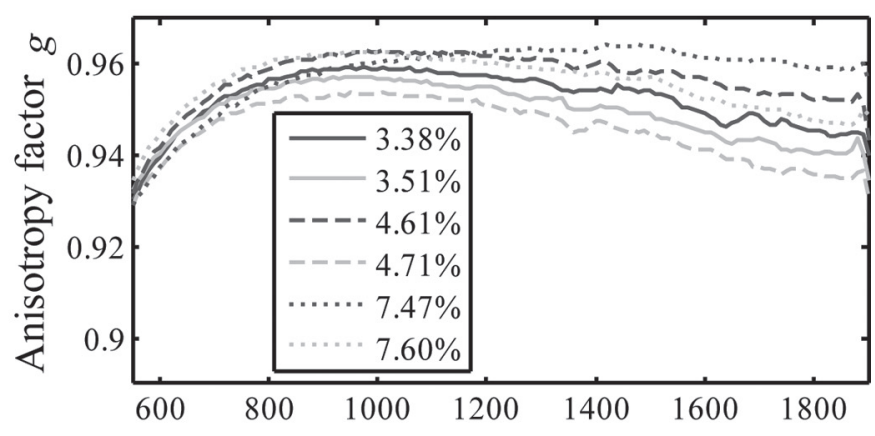

(c)

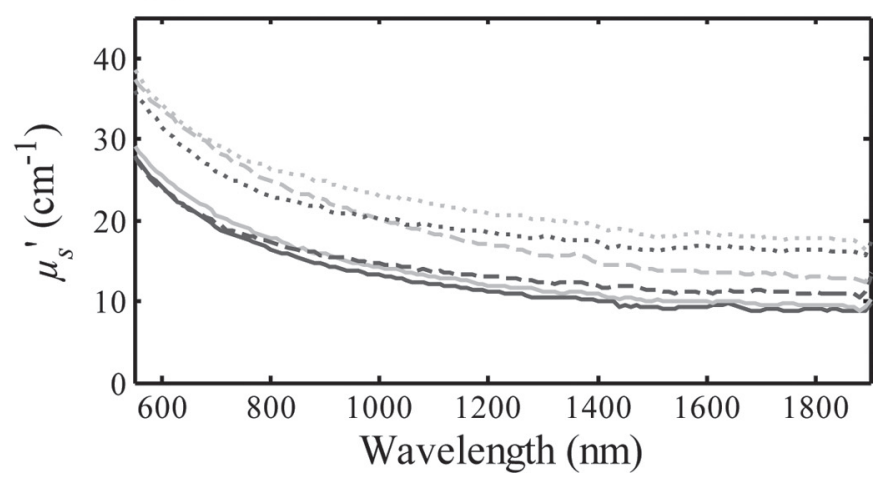

Figure 2. The bulk scattering properties for 6 raw milk samples with an almost constant CP content (2.36-2.49\% vol/vol) and a varying fat content (\% vol/vol in legend): (a) bulk scattering coefficient $\mu_{s}$, (b) anisotropy factor $g$, and (c) the reduced scattering coefficient $\mu_{s}^{\prime}$.

The effect of the fat globule size, which is unambiguously present in the $\mu_{s}$ and $g$ spectra, is not clearly noticeable in the $\mu_{s}^{\prime}$ spectra. Moreover, although the 3 fat content groups could clearly be separated based on their $\mu_{s}$ spectra, only 2 distinct groups appear in the $\mu_{s}^{\prime}$ spectra for wavelengths below 1,200 nm. Furthermore, no grouping of the $\mu_{s}^{\prime}$ spectra can be noticed for wavelengths above $1,200 \mathrm{~nm}$. So, it seems that the fat globule size information present in $\mu_{s}$ and $g$, and the fat content information in $\mu_{s}$ partially neutralize each other if $\mu_{s}$ and $g$ are combined. This implies that $\mu_{s}^{\prime}$ spectra alone might be insufficient to estimate the fat globule size or fat content, or both, and that accurate separation of $\mu_{s}$ and $g$ would be required. This is, however, only feasible if the unscattered transmittance can be accurately measured, or if accurate diffuse reflectance or diffuse transmittance signals, or both, can be collected at very short source-detector distances (Kienle et al., 2001; Sharma and Banerjee, 2003; Prahl, 2010; Kanick et al., 2012; Watté et al., 2012).

As the milk fat globule quantity and size mainly determine the bulk scattering properties, they also have an effect on the measured signals. The diffuse reflectance, total transmittance, and unscattered transmittance spectra for the 6 samples considered in Figure 2 are shown in Figure 3. As $M_{R}$ and $M_{T}$ are the integrated signals over all exit positions and exit angles of the light at, respectively, the reflectance and transmittance side of the sample, the similarity relation is valid and scattering can be described very accurately with only $\mu_{s}^{\prime}$ (Tuchin, 2007; Prahl, 2010). Accordingly, the 2 groups that could be observed from the $\mu_{s}^{\prime}$ spectra [Figure 2(c)] also appear in the $M_{R}$ and $M_{T}$ spectra [Figures 3(a) and 3(b)]. Consequently, the overall levels of the $M_{R}$ and $M_{T}$ spectra do not correlate well with the fat content of the samples. As $\mu_{s}$ is dominant over $\mu_{a}$ in the Vis/NIR range for raw milk, $M_{U}$ is primarily influenced by $\mu_{s}$. This can be clearly observed in Figure 3(c). Moreover, as the $M_{U}$ spectra are presented on a logarithmic scale, the plotted $M_{U}$ spectra are very close to the inverse of the $\mu_{s}$ spectra [Figure 2(a)]. As a result, the 3 fat-content groups also clearly appear in the $M_{U}$ spectra.

\section{Relation Between Bulk Optical Properties and Composition of Raw Milk}

The fat globules and CN micelles are both important absorbing and scattering components in milk. Consequently, the correlation $(\mathrm{r}$ ) between the content of milk fat and CN of all 60 samples (Table 1), and the measured $\mu_{a}$ and $\mu_{s}$ values have been calculated at each of the considered wavelengths (Figure 4). High positive correlation coefficients of 0.751 and 0.632 to 0.762 were found between the fat content $(\% \mathrm{vol} / \mathrm{vol})$ and the $\mu_{a}$ at 1,220 and 1,740 to $1,770 \mathrm{~nm}$. These are most likely related to, respectively, the second and first overtone stretch vibrations of the $\mathrm{CH}_{2}$ bonds (Šašić and Ozaki, 2000). Moreover, a negative correlation ( $\mathrm{r}$ $=-0.616$ ) was found between the fat content and the water absorption at $1,450 \mathrm{~nm}$. As fat is an important part of the DM in milk, a negative correlation with the water content is obvious. The positive correlation ( $\mathrm{r}$ $\geq 0.4$ ) between the fat content and $\mu_{a}$ from 700 until $1,100 \mathrm{~nm}$ cannot be attributed to the absorption by 
fat. Moreover, it might be caused by the small cross talk between $\mu_{s}$ and $\mu_{a}$ [Figure 1(a) detail], as the correlation between the fat content and $\mu_{s}$ in that range is relatively high $[\mathrm{r} \geq 0.839$; Figure 4(b)].

Positive correlations of $0.378,0.157$ to 0.202 , and 0.228 to 0.452 were found between the $\mathrm{CN}$ content $(\%$ $\mathrm{vol} / \mathrm{vol})$ and $\mu_{a}$ at $1,250,1,580$ to 1,620 , and 1,670 to $1,860 \mathrm{~nm}$. These absorption peaks are probably related to respectively the first overtone of amide $\mathrm{A}+$ amide II vibrations, the overlapping first overtone of amide $\mathrm{A}$ and amide B vibrations and the first overtone stretchvibrations of the $\mathrm{CH}$-bonds in the protein side chains (Czarnik-Matusewicz et al., 1999). Similar to fat, CN

(a)

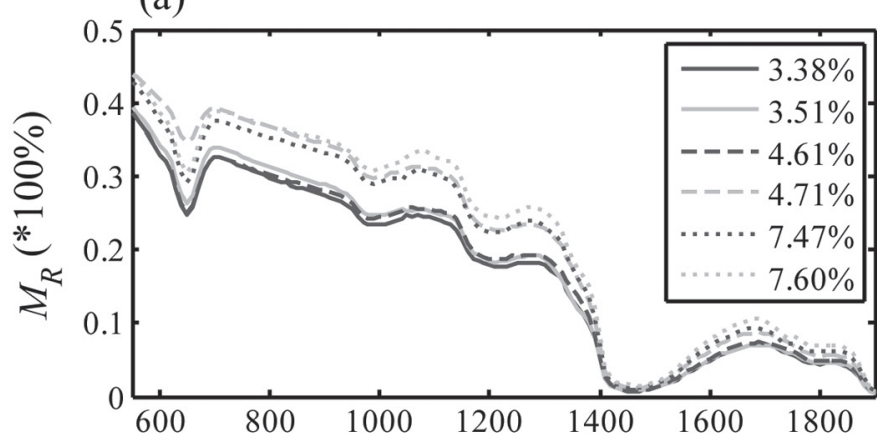

(b)
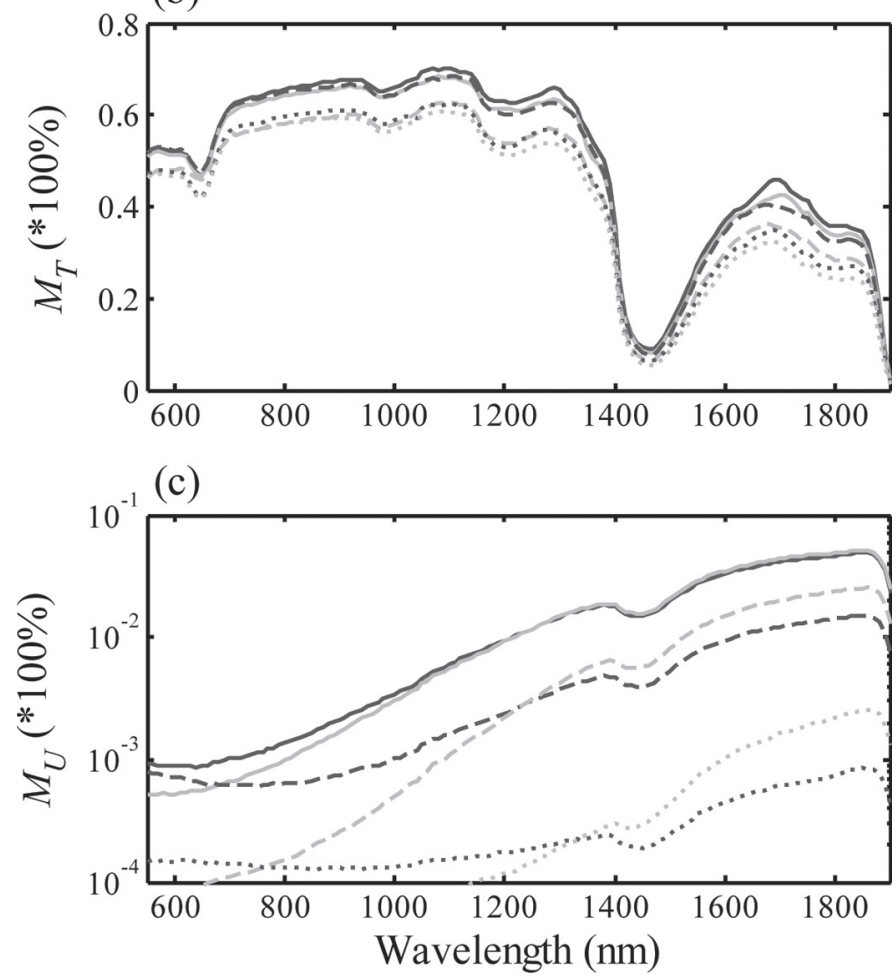

Figure 3. The measured (a) diffuse reflectance $M_{R}$, (b) total transmittance $M_{T}$, and (c) unscattered transmittance spectra $M_{U}$ for 6 raw milk samples with an almost constant CP content $(2.36-2.49 \% \mathrm{vol} /$ $\mathrm{vol}$ ) and a varying fat content (\% vol/vol in legend). followed a negative correlation $(\mathrm{r}=-0.252)$ with the water absorption at 1,450 $\mathrm{nm}$ and an overall positive correlation with $\mu_{a}$ from 700 until 1,100 nm. The latter might also be explained as cross-talk from $\mu_{s}$ to $\mu_{a}$, as in this wavelength range, $\mu_{s}$ has a considerable positive correlation $(\mathrm{r} \geq 0.389)$ with the $\mathrm{CN}$ content [Figure 4(b)]. Around $650 \mathrm{~nm}$, the correlation between $\mu_{a}$ and both the fat and $\mathrm{CN}$ content decreases because the variability in $\mu_{a}$ at these wavelengths is mainly caused by a varying preservative concentration. Compared with milk fat, the CN content has an overall weaker correlation with $\mu_{a}$, which can be explained by the smaller variability of $\mathrm{CP}$ in the analyzed milk samples (Table 1). As the absorption peaks of fat, protein, water, or other milk components (or a combination of these) overlap, it is not possible to get a perfect correlation between the absorption at a single wavelength and the concentration of a milk component. Combination of the absorption information present at different wavelengths through the use of multivariate calibration techniques could help to overcome this selectivity problem. Because almost no scattering effects are present in the $\mu_{a}$ spectra, accurate prediction models could potentially be built on these data without the need for empirical scatter corrections. Moreover, changes in the scattering properties would have (almost) no effect on $\mu_{a}$ such that the predictions are expected to be robust.

In Figure 4(b) the correlation between the $\mu_{s}$ spectra and both the fat and $\mathrm{CN}$ content in raw milk is shown. An overall high positive correlation $(\mathrm{r} \geq 0.766)$ with the fat content ( $\% \mathrm{vol} / \mathrm{vol})$ was found, with the highest correlation ( $\mathrm{r} \geq 0.975$ ) in the 1,300 to $1,400 \mathrm{~nm}$ wavelength range. The very high correlation in the 1,300 to $1,400 \mathrm{~nm}$ region is probably because the size of the fat globules in raw milk has the least effect on $\mu_{s}$ at those wavelengths [Figure 2(a)]. Accordingly, $\mu_{s}$ will be more dependent on the fat content itself. For radiation wavelengths outside the 1,300 to $1,400 \mathrm{~nm}$ range, the size of the fat globules clearly affects $\mu_{s}$, resulting in a lower correlation with the fat content itself.

A much lower correlation $(\mathrm{r}=0.276-0.556)$ was found between the $\mathrm{CN}$ content $(\% \mathrm{vol} / \mathrm{vol})$ and $\mu_{s}$ [Figure 4(b)]. This is probably because CN micelles contribute less to light scattering in the Vis/NIR range (Bogomolov et al., 2012; Aernouts et al., 2015). The correlation, however, increased with decreasing wavelength. Because CN micelles are small $(10-500 \mathrm{~nm})$ compared with the radiation wavelengths $(550-1,900 \mathrm{~nm})$, the $\mu_{s}$ spectrum of CN micelles increases exponentially with decreasing wavelength toward the UV (Aernouts et al., 2015). Moreover, as the native fat globules in raw milk have a size $(0.1-10 \mu \mathrm{m})$ similar to the radiation wavelengths, scattering increases with decreasing wavelength toward a maximum in the Vis/NIR, followed by a decrease 
(a)

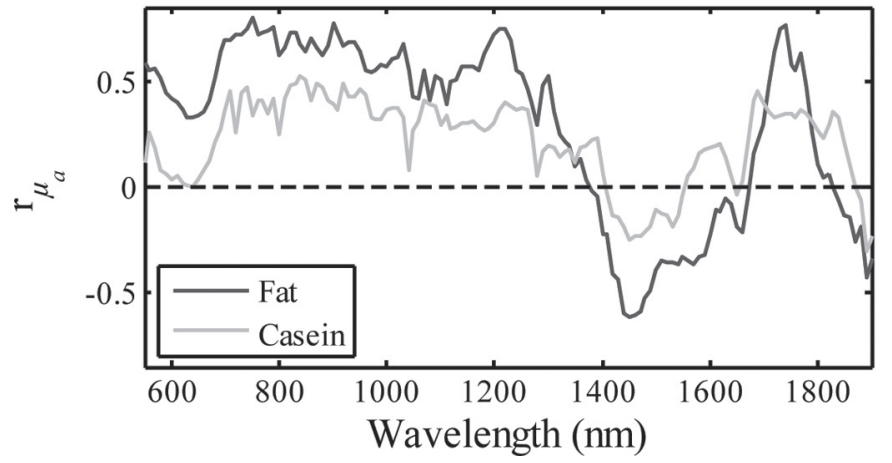

(b)

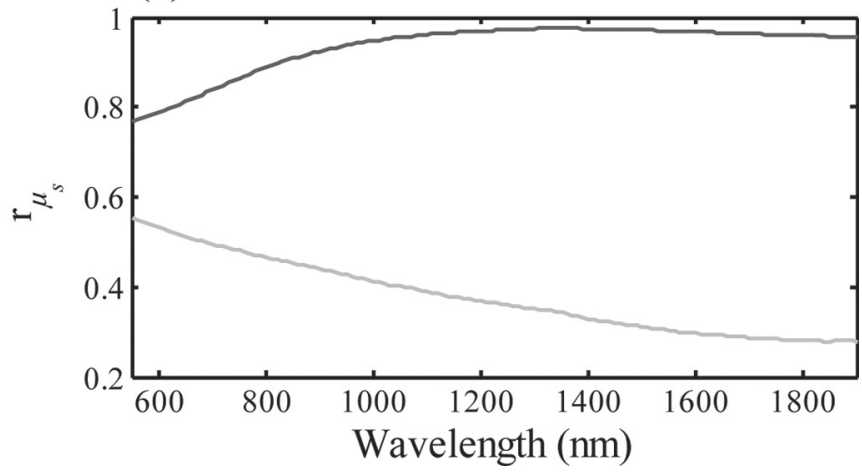

Figure 4. Correlation (r) between the content of milk fat and CN and (a) the bulk absorption coefficient $\mu_{a}$ and (b) the bulk scattering coefficient $\mu_{s}$ in function of the radiation wavelength.

toward the UV [Figure 2(a)]. As a result, the contribution of $\mathrm{CN}$ to the $\mu_{s}$ spectrum of raw milk increases for decreasing wavelengths in the UV/Vis, which confirms the correlations in Figure 4(b). These observations are supported by the findings from other Vis/NIR scattering experiments on raw milk (Bogomolov et al., 2012, 2013; Bogomolov and Melenteva, 2013; Dahm, 2013; Kucheryavskiy et al., 2014). Moreover, it was found that scattering of raw milk at Vis wavelengths near the UV are more related to the CN content, whereas a better relation with the fat content was obtained toward the NIR. This could indicate the potential of UV scattering measurements for estimation of the $\mathrm{CN}$ content in raw milk.

In Figure 5, the relation between the fat content and $\mu_{s}$ at 3 radiation wavelengths $(600,1,300$, and $1,700 \mathrm{~nm})$ is provided. The solid lines in represent the linear fit between the fat content for all 60 raw milk samples and the respective $\mu_{s}$ at each of the 3 considered wavelengths. A poor correlation was found at 600 $\mathrm{nm}$, whereas it was superior around $1,300 \mathrm{~nm}$. This was already clearly indicated in Figure 4(b). The fitted linear lines (solid) generally overestimate the $\mu_{s}$ at low $(<4 \% \mathrm{vol} / \mathrm{vol})$ and high fat concentrations $(>8 \% \mathrm{vol} /$ vol). This indicates that the relation between $\mu_{s}$ and the fat content is probably not linear, resulting from the effect of dependent scattering (Alexander et al., 2002; Gaygadzhiev et al., 2008; Aernouts et al., 2014). An earlier study on milk (Aernouts et al., 2015) showed no significant effect of dependent scattering on the Vis/ NIR $\mu_{s}$ spectra of raw milk if the fat content was below 3 to $4 \%$ (vol/vol). For these raw milk samples, the individual scattering processes will be independent and $\mu_{s}$ is expected to follow a linear increase with increasing fat content if the fat globule size is constant. Nevertheless, if the fat content is above $4 \%$, the scattering fat globules are close enough to influence the scattering by a neighboring fat globule. This generally results in a reduction of the bulk scattering coefficient spectra relative to those expected from the linear independent scattering relations (Alexander et al., 2002; Gaygadzhiev et al., 2008; Aernouts et al., 2014, 2015). To illustrate the effect of dependent scattering, a second linear curve (dashed line) was fitted between the $\mu_{s}$ and the fat content for the raw milk samples with $4 \%$ (vol/vol) fat or less (Figure 5). Compared with the solid line (all data), the dashed line (independent scattering) resulted in a consistently higher slope. Moreover, the linear independent scattering fit (dashed line) generally overestimates the $\mu_{s}$ for fat contents above 4 to $5 \%$ (vol/vol), whereas this effect increases with increasing fat content (Alexander et al., 2002; Gaygadzhiev et al., 2008; Aernouts et al., 2014, 2015). At $600 \mathrm{~nm}$ wavelength, the difference between the slopes of the 2 linear fits is the largest [Figure 5(a)]. This is probably related to the increased variability in $\mu_{s}$ for fixed fat contents [Figure 2(a)], in addition to the effect of dependent scattering. Moreover, the increased variation is likely due to the effect of a varying fat globule size on $\mu_{s}$, which is maximal for radiation wavelengths below 1,100 nm [Figure 2(a)]. Accordingly, the effect of dependent scattering on the difference between slopes is inferior at these wavelengths.

As the effect of dependent scattering on $\mu_{s}$ is clearly present in the data (Figure 5), a nonlinear model, taking into account this effect, would likely result in an improved fit with the data of $\mu_{s}$ versus the fat content (Alexander et al., 2002; Gaygadzhiev et al., 2008; Aernouts et al., 2014). Consequently, measurement of the $\mu_{s}$ at a single wavelength around 1,300 nm could result in very accurate prediction of the fat content in raw milk samples.

The offset of the linear independent scattering fit (dashed line) gives the estimated average $\mu_{s}$ spectrum 
of these samples if no fat globules would be present (Figure 6). Consequently, it relates to the average $\mu_{s}$ spectrum of the $\mathrm{CN}$ fraction in the raw milk samples. In Figure 6, this offset is illustrated in function of the wavelength. Additionally, also the $\mu_{s}$ spectrum simulated for the $\mathrm{CN}$ fraction in a bulk milk sample (fat and $\mathrm{CP}$ content of 4.52 and $2.65 \% \mathrm{vol} / \mathrm{vol}$, respectively), as obtained from a previous study (Aernouts et al., 2015), was plotted. Although the offset spectrum is the result of a fitting procedure on many diverse samples with a variable $\mathrm{CN}$ content (Table 1), fairly good agreement is present between both curves.

(a)

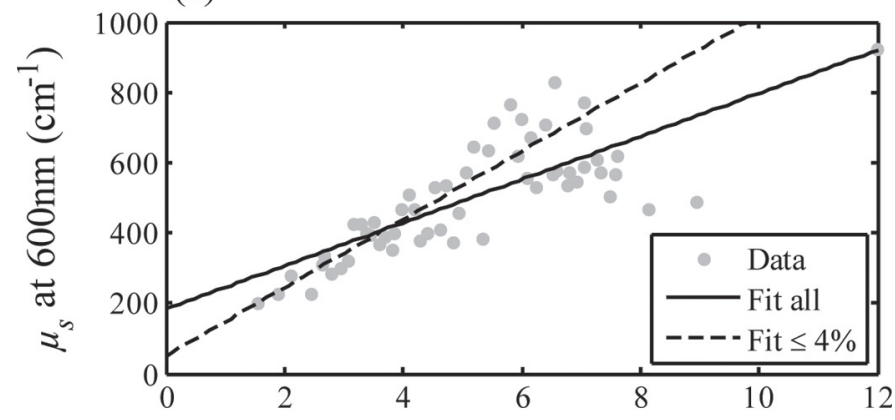

(b)

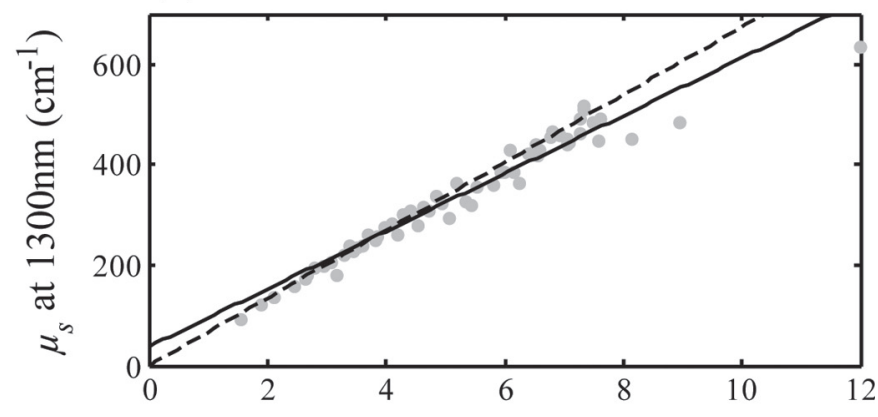

(c)

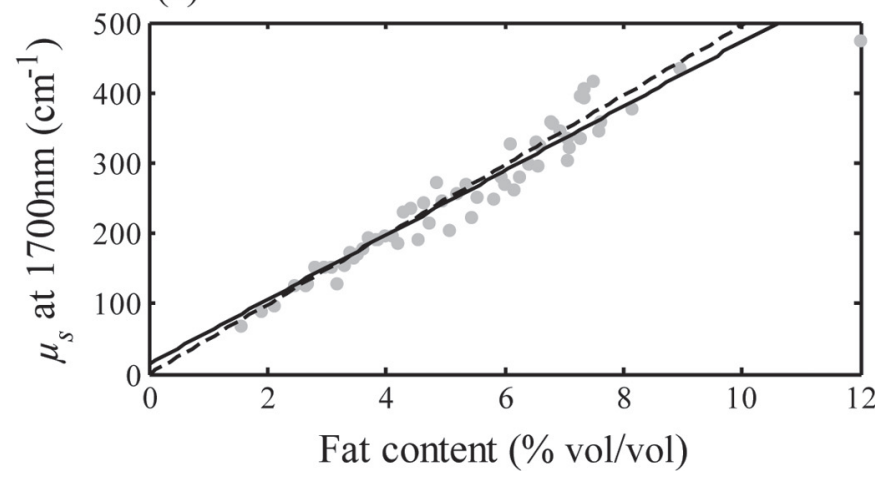

Figure 5. Scatterplots of fat content (\% vol $/ \mathrm{vol})$ versus the bulk scattering coefficient $\mu_{s}$ at (a) $600 \mathrm{~nm}$, (b) 1,300 nm, and (c) 1,700 nm. A linear curve is fitted to all the data points (solid line) and to the data points for which the fat content was below or equal to $4 \%$ (vol/ vol; dashed line).

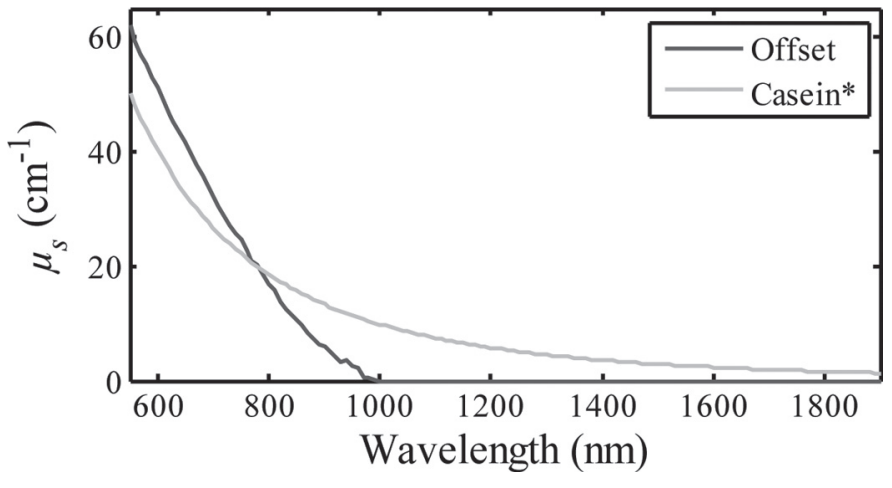

Figure 6. Simulated $(*) \mu_{s}$ spectrum for CN micelle fraction $(2 \%$ $\mathrm{vol} / \mathrm{vol}$ ) in raw milk (Aernouts et al., 2015) and spectrum of offset values for the linear fit between the bulk scattering coefficient $\left(\mu_{s}\right)$ spectra and the fat content for samples with a fat content below $4 \%$ ( $\mathrm{vol} / \mathrm{vol})$.

The obtained information on the BOP of milk can be consulted in simulation studies to improve the insight into Vis/NIR light propagation in milk and other types of emulsions, which is essential for the optimal design of a Vis/NIR spectroscopic sensor that can accurately monitor the quality of raw milk. Moreover, our study indicates that, for the extraction of fat globule size from the scattering properties, a good separation between the bulk scattering coefficients and the anisotropy factors is essential. This can only be achieved with accurate unscattered transmittance measurements or multiple diffuse reflectance or diffuse transmittance measurements close to the point of illumination. Unscattered transmittance measurements of undiluted raw milk is, however, very challenging because very thin path lengths $(<200 \mu \mathrm{m})$ are required, the detector should be installed far $(>1 \mathrm{~m})$ behind the sample, and the unscattered transmittance signals are relatively weak. On the other hand, a small source-detector distance in diffuse reflectance or diffuse transmittance measurements, or both, results in a reduced penetration depth. Accordingly, the minimal distance is limited, as the sampled volume should be representative for the entire sample.

\section{CONCLUSIONS}

The Vis and NIR BOP of a set of 60 raw milk samples representative for milk from Flemish Holstein-Friesian cows have been measured on a double integrating sphere and unscattered transmittance setup. The variation in the absorption coefficient spectra was found to be clearly related to the composition of the milk samples, with clear influences of the water, fat, and CN content. The bulk scattering coefficient spectra were found to be primarily influenced by the quantity and the size of the fat globules. A higher fat content results in an 
overall increase, whereas smaller fat globules produce steeper Vis/NIR bulk scattering coefficient spectra. Accordingly, the observed variation in the Vis/NIR bulk scattering coefficients was large. In the 1,300 to 1,400 $\mathrm{nm}$ wavelength range, the effect of the fat globule size on the bulk scattering coefficient of raw milk was found to be minimal, resulting in a strong positive correlation ( $\mathrm{r} \geq 0.975)$ with the fat content. Moreover, the contribution of the fat content to the bulk scattering coefficient reduced toward the UV, whereas the effect of the CN content increased. The anisotropy factor, on the other hand, is mainly influenced by the size of the fat globules and is almost independent of the particle quantity. Moreover, larger milk fat globules cause more forward scattering of NIR light, which is represented by a higher anisotropy factor. As the fat and CN content had no noticeable effect on the anisotropy factor, the variation in the anisotropy factor spectra of raw milk samples was rather limited. The obtained information on the BOP of milk can be consulted to study the Vis/ NIR light propagation in milk and other types of emulsions. Moreover, this can support the optimal design of a Vis/NIR spectroscopic sensor to accurately monitor the quality of raw milk.

\section{ACKNOWLEDGMENTS}

Ben Aernouts was funded as a Ph.D. fellow of the Research Foundation-Flanders (FWO, Brussels, Belgium, grant 11A4813N). Rodrigo Watté, Robbe Van Beers, and Tjebbe Huybrechts are funded by the Institute for the Promotion of Innovation through Science and Technology in Flanders (IWT-Flanders, Brussels, Belgium, grants 101552,131777 , and 121611 , respectively). The authors gratefully acknowledge IWT-Flanders for the financial support through the GlucoSens project (SB090053).

\section{REFERENCES}

Aernouts, B., E. Polshin, J. Lammertyn, and W. Saeys. 2011. Visible and near-infrared spectroscopic analysis of raw milk for cow health monitoring: Reflectance or transmittance? J. Dairy Sci. 94:5315-5329.

Aernouts, B., R. Van Beers, R. Watté, T. Huybrechts, J. Jordens, D. Vermeulen, T. Van Gerven, J. Lammertyn, and W. Saeys. 2015. Effect of ultrasonic homogenization on the Vis/NIR bulk optical properties of milk: Measurements and simulations. Colloids Surf. B Biointerfaces 126:510-519.

Aernouts, B., R. Van Beers, R. Watté, J. Lammertyn, and W. Saeys. 2014. Dependent scattering in intralipid phantoms in the $600-1850$ nm range. Opt. Express 22:6086-6098.

Aernouts, B., E. Zamora-Rojas, R. Van Beers, R. Watté, L. Wang, M. Tsuta, J. Lammertyn, and W. Saeys. 2013. Supercontinuum laser based optical characterization of turbid media in the $500-2250 \mathrm{~nm}$ range. Opt. Express 21:32450-32467.
Alexander, M., L. F. Rojas-Ochoa, M. Leser, and P. Schurtenberger. 2002. Structure, dynamics, and optical properties of concentrated milk suspensions: An analogy to hard-sphere liquids. J. Colloid Interface Sci. 253:35-46.

Bogomolov, A., S. Dietrich, B. Boldrini, and R. W. Kessler. 2012. Quantitative determination of fat and total protein in milk based on visible light scatter. Food Chem. 134:412-418.

Bogomolov, A., and A. Melenteva. 2013. Scatter-based quantitative spectroscopic analysis of milk fat and total protein in the region $400-1100 \mathrm{~nm}$ in the presence of fat globule size variability. Chemom. Intell. Lab. Syst. 126:129-139.

Bogomolov, A., A. Melenteva, and D. Dahm. 2013. Technical note: Fat globule size effect on visible and shortwave near infrared spectra of milk. J. Near Infrared Spectrosc. 21:435-440.

Cabassi, G., M. Profaizer, L. Marinoni, N. Rizzi, and T. Cattaneo. 2013. Estimation of fat globule size distribution in milk using an inverse light scattering model in the near infrared region. J. Near Infrared Spectrosc. 21:359-373.

Cattaneo, T., G. Cabassi, M. Profaizer, and R. Giangiacomo. 2009. Contribution of light scattering to near infrared absorption in milk. J. Near Infrared Spectrosc. 17:337-343.

Cen, H., R. Lu, and K. Dolan. 2010. Optimization of inverse algorithm for estimating the optical properties of biological materials using spatially-resolved diffuse reflectance. Inverse Probl. Sci. Eng. $18: 853-872$

Czarnik-Matusewicz, B., K. Murayama, R. Tsenkova, and Y. Ozaki. 1999. Analysis of near-infrared spectra of complicated biological fluids by two-dimensional correlation spectroscopy: Protein and fat concentration-dependent spectral changes of milk. Appl. Spectrosc. 53:1582-1594.

Dahm, D. 2013. Review: Explaining some light scattering properties of milk using representative layer theory. J. Near Infrared Spectrosc. 21:323-339.

Erwin, R. E., and H. E. Randolph. 1975. Influence of mastitis on properties of milk. XI. Fat globule membrane. J. Dairy Sci. 58:9-12.

Forsbäck, L., H. Lindmark-Månsson, A. Andrén, M. Akerstedt, L. Andrée, and K. Svennersten-Sjaunja. 2010. Day-to-day variation in milk yield and milk composition at the udder-quarter level. J. Dairy Sci. 93:3569-3577.

Forsbäck, L., H. Lindmark-Månsson, A. Andrén, M. Akerstedt, and K. Svennersten-Sjaunja. 2009. Udder quarter milk composition at different levels of somatic cell count in cow composite milk. Animal 3:710-717.

Friggens, N. C., C. Ridder, and P. Løvendahl. 2007. On the use of milk composition measures to predict the energy balance of dairy cows. J. Dairy Sci. 90:5453-5467.

Frisvad, J. R., N. J. Christensen, and H. W. Jensen. 2007. Computing the scattering properties of participating media using Lorenz-Mie theory. ACM Trans. Graph. 26:60.

Gamm, U. A., S. C. Kanick, H. J. C. M. Sterenborg, D. J. Robinson, and A. Amelink. 2011. Measurement of tissue scattering properties using multi-diameter single fiber reflectance spectroscopy: In silico sensitivity analysis. Biomed. Opt. Express 2:3150-3166.

Gaygadzhiev, Z., M. Corredig, and M. Alexander. 2008. Diffusing wave spectroscopy study of the colloidal interactions occurring between casein micelles and emulsion droplets: Comparison to hard-sphere behavior. Langmuir 24:3794-3800.

Hamann, J., and V. Krömker. 1997. Potential of specific milk composition variables for cow health management. Livest. Prod. Sci. 48:201-208.

ISO (International Organization for Standardization). 2000. Whole milk-Determination of milk fat, protein and lactose contentGuidance on the operation of mid-infrared instruments. International Standard ISO 9622:2000/IDF 141C:2000. International Dairy Federation, Brussels, Belgium.

Kanick, S. C., V. Krishnaswamy, U. A. Gamm, H. J. C. M. Sterenborg, D. J. Robinson, A. Amelink, and B. W. Pogue. 2012. Scattering phase function spectrum makes reflectance spectrum measured from Intralipid phantoms and tissue sensitive to the device detection geometry. Biomed. Opt. Express 3:1086-1100. 
Kaniyamattam, K., and A. De Vries. 2014. Agreement between milk fat, protein, and lactose observations collected from the Dairy Herd Improvement Association (DHIA) and a real-time milk analyzer. J. Dairy Sci. 97:2896-2908.

Katz, G., Z. Schmilovitz, E. Maltz, M. I. Kutscher, M. Sarig, I. Halachmi, A. Hoffman, H. Egozi, and E. Unar, inventors. 2003. Spectroscopic fluid analyzer. Kibutz Afikim (IL), assignee. US Pat. No. 2003/0098969A1.

Katz, G., O. Shapira, L. Lemberskiy-Kuzin, and N. Pinsky, inventors. 2011. System and method for on-line analysis and sorting of milk coagulation properties. S.A.E. Afikim Milking System Agricultural Cooperative Ltd., Kibutz Afikim (IL), assignee. US Pat. No. 8,072,596 B2.

Khankin, D., S. Mordechai, and S. Mark. 2012. Optimization efficiency of Monte Carlo simulation tool for evanescent wave spectroscopy fiber-optic probe. Adv. Opt. Technol. 2012:1-5.

Kienle, A., F. K. Forster, and R. Hibst. 2001. Influence of the phase function on determination of the optical properties of biological tissue by spatially resolved reflectance. Opt. Lett. 26:1571-1573.

Kucheryavskiy, S., A. Melenteva, and A. Bogomolov. 2014. Determination of fat and total protein content in milk using conventional digital imaging. Talanta 121:144-152.

Liu, Q., and N. Ramanujam. 2006. Sequential estimation of optical properties of a two-layered epithelial tissue model from depthresolved ultraviolet-visible diffuse reflectance spectra. Appl. Opt. 45:4776-4790

Logan, A., M. Auldist, J. Greenwood, and L. Day. 2014. Natural variation of bovine milk fat globule size within a herd. J. Dairy Sci. 97:4072-4082.

Løvendahl, P., C. Ridder, and N. C. Friggens. 2010. Limits to prediction of energy balance from milk composition measures at individual cow level. J. Dairy Sci. 93:1998-2006.

Luo, Y., H. Cui, X. Gu, R. Liu, and K. Xu. 2005. Determination of optimal source-detector separation in measuring chromophores in layered tissue with diffuse reflectance. Chin. Opt. Lett. 3:659-661.

McDowell, A. K. R. 1968. Fat testing of composite milk samples with the Milko-tester. J. Dairy Res. 35:181-189.

Melfsen, A., E. Hartung, and A. Haeussermann. 2012. Potential of individual cow scatter correction for an improved accuracy of near infrared milk composition analysis. J. Near Infrared Spectrosc. $20: 477-482$.

Melfsen, A., E. Hartung, and A. Haeussermann. 2013. Robustness of near-infrared calibration models for the prediction of milk constituents during the milking process. J. Dairy Res. 80:103-112.

Michalski, M.-C., B. Camier, V. Briard, N. Leconte, J.-Y. Gassi, H Goudédranche, F. Michel, and J. Fauquant. 2004. The size of native milk fat globules affects physico-chemical and functional properties of Emmental cheese. Lait 84:343-358.

Michalski, M.-C., J.-Y. Gassi, M.-H. Famelart, N. Leconte, B. Camier, F. Michel, and V. Briard-Bion. 2003. The size of native milk fat globules affects physico-chemical and sensory properties of Camembert cheese. Lait 83:131-143.

Mizuno, K., M. Hatsuno, K. Aikawa, H. Takeichi, T. Himi, A. Kaneko, K. Kodaira, H. Takahashi, and K. Itabashi. 2012. Mastitis is associated with IL-6 levels and milk fat globule size in breast milk. J. Hum. Lact. 28:529-534.

Mulligan, F. J., L. O'Grady, D. A. Rice, and M. L. Doherty. 2006. A herd health approach to dairy cow nutrition and production diseases of the transition cow. Anim. Reprod. Sci. 96:331-353.
Nielsen, N. I., T. Larsen, M. Bjerring, and K. L. Ingvartsen. 2005. Quarter health, milking interval, and sampling time during milking affect the concentration of milk constituents. J. Dairy Sci. 88:3186-3200.

Palmer, G., and N. Ramanujam. 2007. Use of genetic algorithms to optimize fiber optic probe design for the extraction of tissue optical properties. IEEE Trans. Biomed. Eng. 54:1533-1535.

Pinsky, N., G. Katz, B. Sabbah, M. I. Kutscher, M. Sarig, Z. Merchav, and A. Gilboa, inventors. 2013. System and method for analyzing fluids. Afikim Agricultural Cooperative Ltd., Kibutz Afikim (IL), assignee. US Pat. No. 8,446,582 B2.

Prahl, S. A. 2010. Everything I think you should know about inverse adding-doubling. Accessed Dec. 20, 2014. http://omlc.ogi.edu/ software/iad/iad-3-9-10.zip.

Šašić, S., and Y. Ozaki. 2000. Band assignment of near-infrared spectra of milk by use of partial least-squares regression. Appl. Spectrosc. 54:1327-1338.

Schenkel, P., R. Samudrala, and J. Hinrichs. 2013. Thermo-physical properties of semi-hard cheese made with different fat fractions: Influence of melting point and fat globule size. Int. Dairy J. 30:7987.

Sharma, D., A. Agrawal, L. S. Matchette, and T. J. Pfefer. 2006. Evaluation of a fiberoptic-based system for measurement of optical properties in highly attenuating turbid media. Biomed. Eng. Online 5:49.

Sharma, S., and S. Banerjee. 2003. Role of approximate phase functions in Monte Carlo simulation of light propagation in tissues. J. Opt. A, Pure Appl. Opt. 5:294-302.

Steponavičius, R., and S. Thennadil. 2009. Extraction of chemical information of suspensions using radiative transfer theory to remove multiple scattering effects: Application to a model two-component system. Anal. Chem. 81:7713-7723.

Steponavičius, R., and S. N. Thennadil. 2011. Extraction of chemical information of suspensions using radiative transfer theory to remove multiple scattering effects: Application to a model multicomponent system. Anal. Chem. 83:1931-1937.

Steponavičius, R., and S. N. Thennadil. 2013. Full correction of scattering effects by using the radiative transfer theory for improved quantitative analysis of absorbing species in suspensions. Appl. Spectrosc. 67:526-535.

Tuchin, V. V. 2007. Tissue Optics: Light Scattering Methods and Instruments for Medical Diagnosis. 2nd ed. SPIE Press, Bellingham, WA.

Vangroenweghe, F., H. Dosogne, and C. Burvenich. 2002. Composition and milk cell characteristics in quarter milk fractions of dairy cows with low cell count. Vet. J. 164:254-260.

Walstra, P., and R. Jenness. 1984. Dairy Chemistry and Physics. John Wiley and Sons, New York, NY.

Walstra, P., J. Wouters, and T. Geurts. 2006. Dairy Science and Technology. 2nd ed. Taylor \& Francis Group, Boca Raton, FL.

Watté, R., B. Aernouts, and W. Saeys. 2012. A multilayer Monte Carlo method with free phase function choice. Proc. SPIE 8429:84290S.

Zamora-Rojas, E., A. Garrido-Varo, B. Aernouts, D. Pérez-Marín, W. Saeys, Y. Yamada, and J. E. Guerrero-Ginel. 2014. Understanding near-infrared radiation propagation in pig skin reflectance measurements. Innov. Food Sci. Emerg. Technol. 22:137-146. 